J. Phys. IV France 127 (2005) 63-68

(C) EDP Sciences, Les Ulis

DOI: $10.1051 /$ jp4:2005127010

\title{
Microscopie interférentielle X-UV de plasmas créés par laser
}

\author{
O. Guilbaud ${ }^{1}$, H. Tang ${ }^{1}$, D. Joyeux ${ }^{2}$, G. Jamelot ${ }^{1}$, A. Klisnick ${ }^{1}$, D. Ros ${ }^{1}$, \\ D. Phalippou ${ }^{2}$, K. Cassou ${ }^{1}$, M. Kado ${ }^{3}$, M. Nishikino ${ }^{3}$, K. Sukegawa ${ }^{3}$, \\ M. Kishimoto ${ }^{3}$, M. Ishino ${ }^{3}$, K. Nagashima ${ }^{3}$ et H. Daido ${ }^{3}$ \\ ${ }^{1}$ LIXAM, Bât. 350, Université Paris-Sud, 91405 Orsay Cedex, France \\ ${ }^{2}$ LCFIO, Institut d'Optique, Bât. 503, Campus d'Orsay, 91403 Orsay Cedex, France \\ ${ }^{3}$ Advanced Photon Research Center, Kansai Research Establishment, JAERI, \\ 8-1 Umemidai, Kizu, Souraku, Kyoto 619-0215, Japan
}

\begin{abstract}
The X-UV interferometry is a promising tools for plasma electron density measurement. We report on the results of such a plasma interferometry experiment using a soft $\mathrm{x}$-ray laser as a probing beam $(\lambda=13.9 \mathrm{~nm})$ and a new X-UV interference microscope. This device is a wavefront division interferometer coupled to a high resolution aspherical imaging mirror. Laser produced plasma electron density profiles were investigated at density as high as $10^{20} \mathrm{~cm}^{-3}$. The expanding plasma has been probed at different times of its evolution. Several nanosecond after its production, the plasma exhibits an index of refraction that is no more below one as expected for a pure plasma. The contribution of the bound electrons of plasma ions to the refractive index is discussed for that situation.
\end{abstract}

\section{INTRODUCTION}

L'interférométrie est une technique couramment utilisée pour mesurer la densité électronique des plasmas. Cette méthode repose sur les propriétés dispersives du milieu étudié dont l'indice de réfraction ne dépend, en première approximation, que de la densité électronique. L'indice de réfraction peut être mesuré en estimant le déphasage qu'introduit le plasma sur un faisceau sonde. Expérimentalement, le plasma est placé sur l'un des bras optiques d'un interféromètre. La comparaison des interférogrammes obtenus avec et sans plasma donne accès à la différence de marche introduite par ce dernier. Une méthode d'inversion appropriée permet de remonter à la distribution spatiale de l'indice de réfraction du plasma et ainsi, à son profil de densité électronique. Théoriquement le faisceau sonde ne peut atteindre que des densités de plasma inférieures à la densité critique $\mathrm{N}_{\mathrm{C}}$, dont la valeur est inversement proportionnelle au carré de la longueur d'onde du faisceau sonde. Au-delà de cette valeur critique, le faisceau sonde est réfléchi. Toutefois, la densité maximale effectivement accessible par la sonde est en pratique très inférieure à $\mathrm{N}_{\mathrm{C}}$. La présence de gradients de densité dans le plasma se traduit par des gradients d'indice de réfraction qui dévient le faisceau sonde hors des zones de grande densité par effet mirage. Cette situation se rencontre fréquemment dans les plasmas produits par interaction laser-cible solide où de forts gradients de densité existent au voisinage de la cible. Au premier ordre, l'angle de déflexion est proportionnel au carré de la longueur d'onde du faisceau sonde. L'apparition de sources X-UV cohérentes, intenses et brèves ainsi que les progrès réalisés sur les optiques X-UV ont ouvert la voie à la réalisation d'expériences d'interférométrie de plasma pouvant potentiellement sonder les régions très denses des plasmas créés par laser [2] [3] [4] [5].

Dans cette perspective, la source X-UV doit présenter plusieurs caractéristiques. La sonde doit être brève afin de figer l'évolution du plasma sondé. Le dispositif de détection des franges étant le plus souvent un détecteur ayant un très long temps de pose (CCD), la source doit émettre un nombre important de photons par unité de surface du champ observée pour surmonter l'émission propre du plasma qui sera également intégrée par le détecteur. La source doit enfin présenter des propriétés de cohérence, spatiale ou temporelle, afin que la production de deux faisceaux mutuellement cohérents au sein de l'interféromètre 
s'effectue avec un rendement élevé. Des expériences préliminaires d'interférométrie de plasma ont ainsi été réalisées avec des harmoniques d'ordre élevé [2], des lasers X-UV quasi-stationnaires [4], à décharge capillaire [5] et à pompage transitoire[3]. Le laser X-UV à pompage transitoire [1] pourrait constituer un compromis intéressant entre les sources ultrabrèves $(\sim \mathrm{fs})$ mais peu énergétiques que sont les harmoniques et les lasers X-UV quasistationnaires, très énergétiques mais émettant des impulsions X-UV relativement longues $(\sim 100 \mathrm{ps})$.

\section{DISPOSITIF EXPÉRIMENTAL}

Nous présentons les résultats d'une expérience d'interférométrie de plasma employant un laser XUV à pompage transitoire à argent nickelloïde, émettant des impulsions X-UV monochromatiques à $\lambda=13.9 \mathrm{~nm}$ [13]. Cette source est développée à l'Advanced Photon Research Center (Nara, Japon) [14]. Elle délivre des impulsion de quelques $\mu \mathrm{J}$ d'énergie durant quelques picosecondes. La divergence du faisceau est de $5 \mathrm{mrad}$ par 10mrad pour une taille de source de $50 \mu \mathrm{m}$ par $10 \mu \mathrm{m}$. Une chaîne laser CPA Néodyme-verre est utilisée pour générer ce laser X-UV. Dans ce schéma de pompage, une impulsion laser non comprimée $\left(600 \mathrm{ps}, 10^{12}\right.$ W.cm $\left.{ }^{-2}\right)$ crée un plasma contenant les ions argents nickelloïdes à partir d'une cible d'argent solide. Cette impulsion est suivie 1,2 ns plus tard d'une impulsion laser comprimée $\left(1 \mathrm{ps}, 10^{15} \mathrm{~W} . \mathrm{cm}^{-2}\right)$ qui chauffe les électrons du plasma préformé à une température de plusieurs centaines d'eV. Les collisions électrons - ions créent une inversion de population sur une transition 4d-4p de l'ion argent nickelloïde responsable du gain laser dans le plasma.

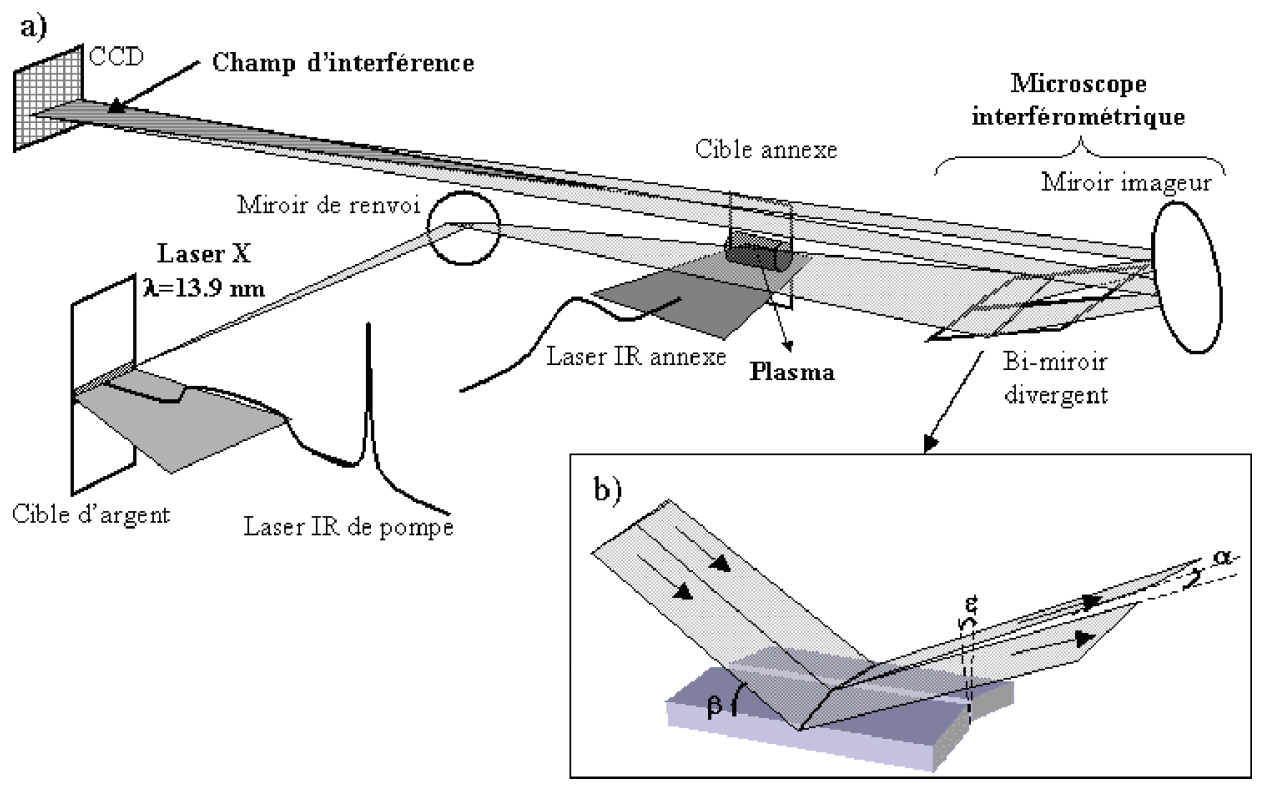

Figure 1. a) dispositif expérimental. b) schéma de principe du bi-miroir divergent de Fresnel.

La figure 1.a présente le dispositif expérimental employé. Un miroir de renvoi multicouche dirige le faisceau laser X-UV vers un plasma «échantillon ». Celui-ci est produit en focalisant une impulsion laser IR non-comprimée annexe (1 J, 1,6 ns) sur une cible solide d'aluminium. La surface de la cible est parallèle à l'axe de propagation du laser X. Le laser infrarouge est focalisé selon une ligne focale d'axe parallèle à l'axe du laser $X$. Le plasma ainsi produit est filiforme $(100 \mu \mathrm{m} \times 1 \mathrm{~mm}$ pour une ligne focale de $100 \mu \mathrm{m} \times 9 \mathrm{~mm}$ ) et ses propriétés peuvent être considérées comme étant invariante le long de l'axe de propagation du faisceau laser X-UV. Cette géométrie a été adoptée pour faciliter l'inversion des interférogrammes. 
Après avoir traversé le plasma, le faisceau est collecté par un microscope interférométrique X-UV, développé par le Laboratoire Charles Fabry de l'Institut d'Optique [8]. Il se compose de deux éléments optiques : un bi-miroir de Fresnel suivi d'un miroir imageur ellipsoïdal. Le bi-miroir est constitué de deux miroirs fonctionnant en incidence rasante et formant un toit dont l'arête est orientée suivant l'axe du faisceau laser X-UV(figure 1.b). Après réflexion sur le bi-miroir, le faisceau X-UV incident est séparé en deux sous-faisceaux. Le premier sous-faisceau correspond à la portion du faisceau incident qui a traversé le plasma et sera appelé « faisceau sonde ». Le second sous-faisceau correspond quant à lui à la portion du faisceau incident qui est passée loin du plasma. Il joue le rôle d'un faisceau de « référence ».

Le miroir imageur forme l'image du plan de sortie du plasma échantillon sur un détecteur (CCD XUV Princeton) avec un grandissement de 12. Le forme du miroir est proche de la forme d'un ellipsoïde à mieux que $0,5 \mathrm{~nm}$ rms [6]. Les aberrations géométriques sont ainsi fortement minimisées, de telle sorte que la résolution d'un tel dispositif est limitée par la diffraction. La résolution maximale de cet instrument est de l'ordre de $1 \mu \mathrm{m}$ dans le plan objet. Toutefois, le grandissement choisi et la taille des pixels de la caméra employée n'autorise ici qu'une résolution maximale de $2 \mu \mathrm{m}$. Le miroir imageur assure la recombinaison des deux sous-faisceaux, sonde et référence, sur le plan du détecteur où ils interfèrent. Autrement dit, le bi-miroir génère des interférences virtuelles dans la région du plasma qui est imagée par le miroir asphérisé.

Le champ des interférences dans le plan objet est de $2 \mathrm{~mm}$ par $500 \mu \mathrm{m}$ avec un interfrange de $10 \mu \mathrm{m}$. Les franges sont contrastées mais leur amplitude est faible. Deux facteurs abaissent ce niveau de signal. Tout d'abord, l'interféromètre est placé loin de la source ( $3 \mathrm{~m}$ ). Ainsi seule une petite portion du faisceau laser X est utilisée par l'interféromètre, cela afin de bénéficier d'une cohérence spatiale suffisante pour réaliser des interférences. Enfin, le grandissement $\mathrm{g}$ du dispositif imageant diminue le nombre de photon par unité de surface du détecteur par un facteur $\mathrm{g}^{2}=144$.

\section{ANALYSE DES INTERFÉROGRAMMES}

La figure 2.a présente un interférogramme typique obtenu en présence de plasma. Le détecteur intègre non seulement l'interférogramme laser X-UV, mais également l'image intégrée en temps de l'émission propre du plasma (à la longueur d'onde du laser X-UV). Cette émission est superposée à l'interférogramme et peut dans certains cas le masquer. Ainsi, près de la surface de la cible, l'émission propre du plasma peut

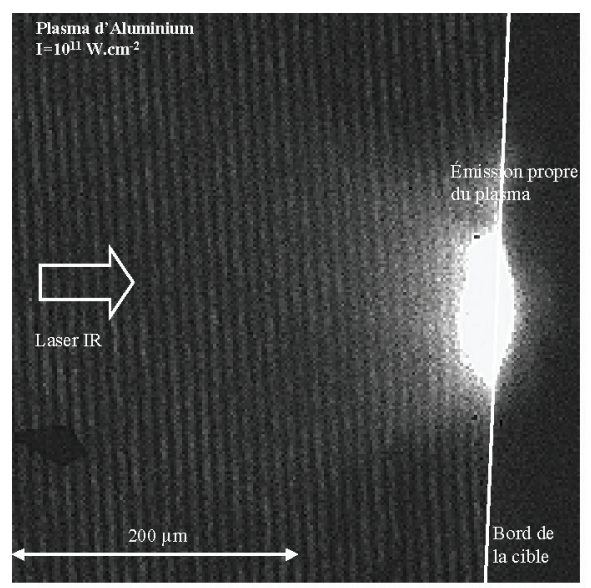

a)

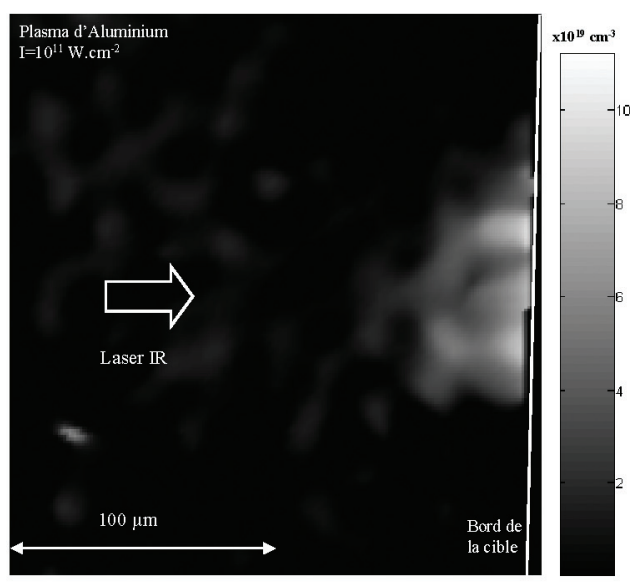

b)

Figure 2. a) interférogramme obtenu en présence de plasma. La ligne blanche représente le bord de la cible. La zone brillante correspond à l'émission propre du plasma. A proximité de cette zone les franges sont déviées vers la cible b) Carte de densité électronique déduite de l'interférogramme précédent. 
être jusqu'à cinquante fois supérieure à l'amplitude de la modulation des franges. Toutefois, la caméra ne sature pas et sa dynamique très élevée permet de suivre cette modulation jusqu' à $10 \mu \mathrm{m}$ de la surface de la cible. Près de la zone fortement émettrice, qui n'est autre que la zone d'interaction du laser IR avec la cible, les franges son déviées vers la cible. Ceci est la manifestation de l'indice de réfraction du plasma traversé par le faisceau sonde. La mesure du déplacement des franges permet d'accéder à la densité électronique locale.

La traduction du déplacement local des franges en une carte de densité électronique s'effectue en plusieurs étapes. Après un filtrage des basses fréquences spatiales de l'image, on effectue une transformée de Fourier de cette dernière. Le signal des franges apparaît alors dans l'espace de Fourier comme une fonction centrée sur la fréquence spatiale associée à la modulation des franges. Un filtrage autour de cette fréquence permet d'isoler en partie le signal porté par la modulation des franges des autres signaux parasites (fond, émission propre du plasma). Plus la bande passante du filtre sera fine, plus grande sera la perte d'information sur la modulation des franges. Ceci se traduira en particulier par une diminution de la résolution spatiale sur le profil de densité. Un compromis entre réjection des signaux parasites et résolution spatial doit être trouvé selon le rapport signal à bruit. Un traitement supplémentaire du spectre spatial permet d'obtenir la carte de phase de la modulation des franges. Cette carte de phase est finalement convertie en une carte d'indice de réfraction puis de densité électronique (figure 2.b).

L'analyse des interférogrammes obtenus montre que la technique employée permet de sonder des densités de l'ordre de $10^{20}$ électrons par $\mathrm{cm}^{-3}$ à moins de $10 \mu \mathrm{m}$ de la surface initiale de la cible (plasma d'aluminium sondés 1 ns après l'arrivée du pic de l'impulsion laser IR sur la cible). Des calculs de tracé de rayons montrent a posteriori que la réfraction du faisceau laser $\mathrm{X}$ par les gradients de densité mesurés est négligeable. La résolution spatiale de ces profils de densité est limité par la bande passante du filtre numérique employé dans le traitement d'image. Elle est ici de $30 \mu \mathrm{m}$ au maximum. Pour améliorer cette résolution spatiale, un meilleur rapport signal laser X-UV sur signal de l'émission propre est nécessaire.

\section{DISCUSSION}

La durée de l'impulsion laser X, de l'ordre de quelques picosecondes [7], est beaucoup plus courte que le temps caractéristique d'évolution du plasma. Les interférogrammes permettent ainsi d'obtenir le profil de densité instantané du plasma. En faisant varier le délai entre l'impulsion laser IR créant le plasma et l'impulsion laser X-UV de sonde, il a été possible de reconstituer l'évolution de la densité électronique au cours de l'expansion du plasma. L'effet de l'intensité de l'impulsion laser IR sur le profil de densité a pu également être étudié en changeant l'énergie du faisceau infrarouge.

Pour des délais importants ( 2 à 3 ns après le pic d'intensité du laser annexe), on observe près de la surface de la cible, une inversion du sens de déplacement des franges, et ce, pour des intensités du

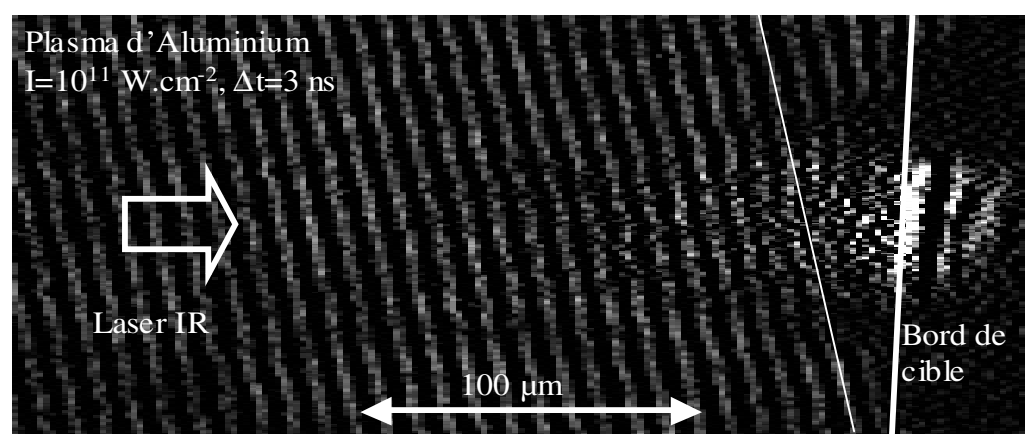

Figure 3. Inversion du sens de déplacement des franges. Interférogramme de plasma d'aluminium réalisé 3 ns après l'arrivée de l'impulsion laser IR sur la cible. Près de la cible les franges sont décalées vers le vide. 
laser annexe variant entre $2.10^{9}$ et $10^{11} \mathrm{~W} \cdot \mathrm{cm}^{-2}$. Ce phénomène, également observé par l'équipe de $\mathrm{J}$. Dunn et al. [12], ne peut être interprété par l'apparition d'un creux de densité près de la cible car celui-ci se traduirait par un déplacement des franges nul à cet endroit. Une inversion du sens de déplacement des franges correspond à un changement de signe de la différence de marche $\delta=(\mathrm{n}-1) \ell$. Pour un plasma fortement ionisé, la polarisabilité est dominée par la contribution des électrons libres. L'indice de réfraction est alors inférieur à un. Si la contribution des électrons liés à l'indice de réfraction n'est plus négligeable, ce dernier peut devenir supérieur à un et conduire à un changement de signe de $\delta$. Une situation extrême est celle d'un plasma suffisamment refroidi pour redevenir une vapeur d'aluminium (absence d'électrons libres). L'indice de réfraction de cette vapeur peut être calculé à partir des coefficients complexes de diffusion atomique [11]. Pour une densité particulaire de $10^{20}$ atomes d'aluminium par $\mathrm{cm}^{-3}$ la valeur de l'indice de réfraction d'une vapeur d'aluminium est compatible avec le sens et l'amplitude du déplacement des franges observés. Toutefois, l'absorption correspondante serait telle qu'aucune frange ne devrait être observable (seuil L3 de l'aluminium). Le milieu sondé à cet instant n'est donc pas une vapeur d'aluminium mais plus probablement un plasma faiblement ionisé. En effet, l'intensité laser sur la cible est faible et l'inversion des franges est observée pour des délais très longs, pour lesquels le plasma n'est plus chauffé par l'impulsion laser. Un tel milieu peut présenter un indice $\mathrm{n}>1$, en particulier si l'un des ions présents dans le plasma possède une raie de résonance de fréquence proche de celle du faisceau sonde. L'aluminium (III) possède une telle raie $\left(2 \mathrm{p}^{6} 3 s-2 \mathrm{p}^{5} 3 s 3 d^{2} \mathrm{~S}-{ }^{4} \mathrm{D}, \lambda=13.883 \mathrm{~nm}, \mathrm{f}_{\mathrm{osc}}=0.37\right)$ [10]. Cette interprétation est confortée par des calculs récents effectués par Nilsen [9] qui soulignent en particulier l'effet dominant de réfraction anormale par la raie de résonance de l'aluminium (III). Dans cette situation, l'estimation de la densité électronique du plasma à partir du déplacement des franges est rendue très difficile. Il faut connaître les populations ioniques du plasma en un point et en un instant donné puis calculer la contribution de chaque type d'ion à l'indice de réfraction global. Par ailleurs, la présence d'ions Al (III) dès les premiers instants d'existence du plasma pourrait conduire à une sous-estimation de la densité électronique réelle du plasma. Une comparaison des résultats expérimentaux aux résultats de codes hydrodynamiques est nécessaire pour quantifier cet écart.

\section{CONCLUSION}

Nous avons utilisé un laser X-UV à pompage transitoire pour sonder des plasmas d'aluminium créés par laser. Un interféromètre X-UV à division de front d'onde a été employé en association avec un miroir imageur X-UV de $2 \mu \mathrm{m}$ de résolution spatiale pour déterminer le profil de densité électronique du plasma. Des densités électroniques de $10^{20}$ électrons par $\mathrm{cm}^{-3}$ ont pu ainsi être mesurées. L'énergie de la source employée comparée à celle émise par le plasma détermine les performances de cette technique, notamment en terme de résolution spatiale. Au cours de cette expérience l'effet des électrons liés à l'indice de réfraction du plasma a été mise en évidence de façon spectaculaire. Il apparaît que pour des plasmas faiblement ionisés la détermination de la densité électronique du plasma devient un problème complexe. Cependant, pour des plasmas fortement ionisés, les calculs montrent que ce problème est moins critique.

\section{Remerciements}

Ce travail a été réalisé dans le cadre du contrat PICS Nº1631.

\section{Bibliographie}

[1] P.V. Nickles, V.N. Shlyaptsev, M.P. Kalachnikov, M. Schnürer, I. Will, W. Sandner, Phys. Rev. Lett. 78, (1997) p 2748

[2] P. Salières, L. Le Déroff, T. Auguste, P. Monot, P. d'Oliveira, D. Campo, J.-F. Hergott, H. Merdji, B. Carré, Phys. Rev. Lett. 83 (1999), p 5483-5486 
[3] R.F. Smith, J. Dunn, J. Nilsen, V.N. Shlyaptsev, S. Moon, Filevich, J.J. Rocca, M.C. Marconi, J.R. Hunter, T.W. Barbee Jr, Phys. Rev. Lett. 8965004 1-4 (2002)

[4] L.B. DaSilva, T.W. Barbee Jr, et al., Phys. Rev. Lett. 74 (1995) pp 3991-3994

[5] J. Filevich, K. Kanizay, M.C. Marconi, J.L.A. Chilla, and J.J. Rocca, Optics Lett. 25, 356 (2000)

[6] D. Joyeux, D. Phalippou, R. Mercier, M. Mullot, M. Lamare, A. Klisnick, D. Ros, O. Guilbaud, G. Jamelot, J. Phys IV France, pp 263-266

[7] A. Klisnick, J. Kuba, D. Ros, R. Smith, G. Jamelot, C. Chenais-Popovics, R. Keenan, S.J. Toppings, C.L.S. Lewis, F. Strati, G.J. Tallents, D. Neely, R. Clarke, J. Collier, A.G. MacPhee, F. Bartolotto, P.V. Nickles and K.A. Janulewicz, Phys. Rev. A 65, 033810 (2002)

[8] S. Hubert, Interférométrie de plasma à l'aide de lasers X-UV, (thèse de doctorat, Université Paris-Sud, 2001)

[9] J. Nilsen et al., Applications of X-ray lasers utilizing plasmas that are only a few times ionized, 9th International Conference on X-ray Lasers, may 2004, Beijing, China, to be published

[10] J.B. West et al., Phys. Rev. A, 63, p 052719

[11] E.M. Gullikson, P. Denham, S. Mrowka, J.H. Underwood, Phys. Rev. B, 49 (1994), p 16283-8

[12] J. Dunn et al., Applications of X-ray lasers utilizing plasmas that are only a few times ionized, 9 th International Conference on X-ray Lasers, may 2004, Beijing, China, to be published

[13] H. Tang, O. Guilbaud, G. Jamelot, D. Ros, A. Klisnick, D. Joyeux, D. Phalippou. M. Kado, M. Nishikino, M. Kishimoto, K. Sukegawa, M. Ishino, K. Nagashima and H. Daido, Applied Physics B, 78 (2004) pp 975-977

[14] Y. Kato et al., X-ray laser developpment at Advanced Photon Research Center, 7th International Conference on X-ray Lasers, may 200, St Malo, France, G. Jamelot, C. Möller, A. Klisnick Eds. (J. Phys IV, France, 11, 2001) 